\title{
Correlación de los valores de sFlt-1, PlGF y su cociente en preeclampsia con criterios de severidad en una Unidad de Cuidados Intensivos**
}

Correlation of the values of sFlt-1, PlGF and its precilampsy court with severity criteria in an Intensive Care Unit

Correlação dos valores de sFlt-1, PlGF e seu coeficiente em preeclampsia com critérios de gravidade em uma Unidade de Terapia Intensiva

Luis Alberto Ruiz Marines, ${ }^{*}$ José Antonio Hernández Pacheco, ${ }^{*}$ Virginia Medina Jiménez, ${ }^{*}$ Lida Montserrat Cruz Gómez, Dulce María Bertha Uicab Jiménez, ${ }^{*}$ Miguelina de Jesús Cruz López, ${ }^{*}$ Guillermo Garduño García*

\section{RESUMEN}

Introducción: La detección del desequilibrio entre los factores proangiogénicos/antiangiogénico (sFIt-1, PIGF, cociente sFIt-1/PIGF) en la sangre materna son herramientas de pronóstico y diagnóstico en preeclampsia.

Objetivo: Determinar la correlación entre los valores sanguíneos de sFlt-1, PIGF, cociente sFIt-1/PIGF y las complicaciones en mujeres con preeclampsia severa.

Material y métodos: Se estudiaron a mujeres que ingresaron a la $\mathrm{UCI}$ con diagnóstico de preeclampsia con criterios de severidad y se determinaron las variables clínicas y de laboratorio. Las concentraciones séricas de sFlt-1 y PIGF se realizaron con un equipo automático KRYPTOR compact Plus.

Resultados: Encontramos que $33.3 \%$ fue preeclampsia temprana y $66.7 \%$ tardía. Los criterios de severidad fueron: $66.7 \%$ crisis hipertensiva y $33.3 \%$ encefalopatía hipertensiva. Existió una correlación negativa entre los valores de sFlt-1 y urea, creatinina, proteínas de orina en 24 horas, presión sistólica (TAS) y presión diastólica (TAD). La correlación fue pobre y no fue estadísticamente significativa. Existió una correlación positiva y estadísticamente significativa para ácido úrico. Existió una correlación negativa entre los valores de PIGF en TAS, TAD. No existió correlación entre los valores cociente sFIt-1/PIGF y las variables medidas como TAS, TAD.

Conclusiones: Este estudio confirma que es posible identificar un desbalance angiogénico en mujeres con preeclampsia severa. Sin embargo, los marcadores angiogénicos no presentaron una correlación estadísticamente significativa con respecto a las variables clínicas y bioquímicas de preeclampsia en la Unidad de Cuidados Intensivos.

Palabras clave: Preeclampsia, terapia intensiva obstétrica, sFlt-1, PIGF, factores angiogénicos.

\section{ABSTRACT}

Introduction: The detection of the imbalance between proangiogenic/ antiangiogenic factors (sFlt-1, PIGF, sFIt-1/PIGF ratio), in maternal blood are prognostic and diagnostic tools in preeclampsia.

Objective: To determine the correlation between blood values of (sFlt-1, PIGF, sFlt-1/PIGF ratio) and complications in women with severe preeclampsia. Material and methods: Women who were admitted to the ICU with a diagnosis of preeclampsia with severity criteria were studied, clinical and laboratory variables were determined. Serum concentrations of sFlt-1, PLGF were performed with a KRYPTOR compact Plus automatic equipment.

Results: $33.3 \%$ were early preeclampsia and $66.7 \%$ late. The severity criteria occurred with $66.7 \%$ with hypertensive crisis and $33.3 \%$ with hypertensive encephalopathy. There was a negative correlation between the values of sFlt-1 and urea, creatinine, urine proteins in 24 hours, systolic pressure (ASD), diastolic pressure (ADT). The correlation was poor and not statistically significant. There was a positive and statistically significant correlation for uric acid. There was a negative correlation between PIGF values in TAS, TAD. There was no correlation between the sFlt-1/PIGF quotient values and the variables measured as TAS, TAD. Conclusions: This study confirms that it is possible to identify an angiogenic imbalance in women with severe preeclampsia. However, the angiogenic

** Ganador del Premio Académico «Dr. Mario Shapiro» 2019. Tercer lugar.

\footnotetext{
* Instituto Nacional de Perinatología.
}

Abreviaturas: sFlt-1 = Soluble fms-like tyrosine kinase-1. PIGF = Placental Growing Factor. $\mathrm{UCI}=$ Unidad de Cuidados Intensivos. TGO = Transaminasa Glutámico-Oxalacética. TGP = Alanina-aminotransferasa. $\mathrm{SDG}=$ Semanas de gestación .

Recepción: 30/08/2019. Aceptación: 03/12/2019.

Este artículo puede ser consultado en versión completa en www.medigraphic.com/medicinacritica markers did not show a statistically significant correlation, with respect to the clinical and biochemical variables of preeclampsia in the Intensive Care Unit. Keywords: Preeclampsia, intensive obstetric therapy, sFIt-1, PIGF, angiogenic factors.

\section{RESUMO}

Introdução: A detecção de desequilíbrio entre fatores pró-angiogênicos/ antiangiogênicos (sFlt-1, PIGF, coeficiente sFlt-1/PIGF) no sangue materno são ferramentas de prognóstico e diagnóstico na pré-eclâmpsia.

Objetivo: Determinar a correlação entre os valores sanguíneos de (sFlt-1, PIGF, coeficiente sFlt-1/PIGF) e complicações em mulheres com pré-eclâmpsia grave.

Material e métodos: Foram estudadas mulheres admitidas na UTI com diagnóstico de pré-eclâmpsia com critérios de gravidade, determinou-se variáveis clínicas e laboratoriais. As concentrações séricas de sFlt-1, PLGF foram realizadas com um equipamento automático KRYPTOR compact Plus.

Resultados: $33.3 \%$ eram pré-eclâmpsia precoce e $66.7 \%$ tardia. Os critérios de gravidade ocorreram com $66.7 \%$ com crise hipertensiva e $33.3 \% \mathrm{com}$ encefalopatia hipertensiva. Houve correlação negativa entre os valores de sFlt-1 e uréia, creatinina, proteínas da urina em 24 horas, pressão sistólica (PAS) e pressão diastólica (PAD). A correlação foi ruim e estatisticamente não significante. Houve uma correlação positiva e estatisticamente significante para o ácido úrico. Encontrou-se uma correlação negativa entre os valores de PLGF no TAS, TAD. Não houve correlação entre os valores do quociente sFlt-1/PIGF e as variáveis medidas como PAS e $P A D$.

Conclusões: Este estudo confirma que é possível identificar um desequilíbrio angiogênico em mulheres com pré-eclâmpsia grave. No entanto, os marcadores angiogênicos não apresentaram correlação estatisticamente significante com relação às variáveis clínicas e bioquímicas da pré-eclâmpsia na unidade de terapia intensiva.

Palavras-chave: Pré-eclâmpsia, terapia intensiva obstétrica, sFlt-1, PIGF, fatores angiogênicos.

\section{INTRODUCCIÓN}

La detección del desequilibrio entre los factores proangiogénicos/antiangiogénico (sFlt-1, PIGF y cociente sFIt-1/PIGF) en la sangre materna es una herramienta de pronóstico y diagnóstico en preeclampsia. ${ }^{1,2}$ Diversos autores han encontrado resultados variables en la correlación entre la medición sanguínea de estos factores y las complicaciones que ocurren en la preeclampsia severa. ${ }^{3,4}$ En una revisión en la literatura no encontramos estudios que repitieran estos hallazgos en humanos. El objetivo del presente estudio es determinar la correlación entre los valores sanguíneos de sFlt1, PIGF, cociente sFIt-1/PIGF y las complicaciones en mujeres con preeclampsia severa. ${ }^{2}$

\section{MATERIAL Y MÉTODOS}

Se estudiaron a mujeres que ingresaron a la UCl del Instituto Nacional de Perinatología «Isidro Espinosa de los 
Reyes» con diagnóstico de preeclampsia con criterios de severidad, y de cualquier edad gestacional y materna («grupo 1»). ${ }^{5-7}$ Con la finalidad de comparar los valores del equilibrio angiogénico, cada mujer con preeclampsia se pareó por edad gestacional y edad materna con mujeres sanas («grupo 2»). En todos los casos se determinaron las variables clínicas y de laboratorio; para la medición en sangre materna, se tomaron $5 \mathrm{~mL}$ de sangre periférica en un tubo y sin anticoagulante, se centrifugó a 3,200 RPM durante 10 minutos. Las concentraciones séricas de sFlt-1 y PIGF se realizaron con un equipo automático KRYPTOR compact Plus, BRAHMS-Instruments y se reportaron en $\mathrm{ng} / \mathrm{mL}$. El radio sFlt-1/PIGF se obtuvo mediante la división simple de los dos valores. Como análisis secundario se determinó la correlación entre los valores del equilibrio angiogénico con los criterios de severidad. El análisis estadístico incluyó las medidas de tendencia central y proporciones, así como medidas de dispersión. Se estimó la correlación de Pearson por medio del programa SPSS 23.0 considerando como estadísticamente significativo un valor de $p<0.05$.

\section{RESULTADOS}

Se obtuvo un total de 42 mujeres en el «grupo 1 », y de 22 en el «grupo 2». La edad promedio entre el grupo 1 y

Tabla 1: Correlaciones de sFIt-1, PIGF y sFlt-1/ PIGF y variables clínico-bioquímicas.

\begin{tabular}{|c|c|c|}
\hline Variables & $\begin{array}{l}\text { Correlación de } \\
\text { Pearson (r) }\end{array}$ & $\mathrm{p}$ \\
\hline sFlt-1 vs TAS & -0.188 & 0.32 \\
\hline sFlt-1 vs TAD & -0.11 & 0.564 \\
\hline sFlt-1 vs urea & -0.119 & 0.53 \\
\hline sFlt-1 vs creatinina & -0.156 & 0.41 \\
\hline sFlt-1 vs ácido úrico & 0.369 & 0.045 \\
\hline sFlt- 1 vs plaquetas & -0.003 & 0.989 \\
\hline sFlt-1 vs depuración de creatinina & -0.014 & 0.947 \\
\hline sFlt-1 vs proteínas de 24 horas & -0.146 & 0.467 \\
\hline sFIt-1 vs proteínas/creatinina & -0.227 & 0.557 \\
\hline PIGF vs TAS & -0.299 & 0.108 \\
\hline PIGF vs TAD & -0.138 & 0.466 \\
\hline PIGF vs urea & 0.101 & 0.596 \\
\hline PIGF vs creatinina & 0.179 & 0.345 \\
\hline PIGF vs ácido úrico & 0.104 & 0.583 \\
\hline PIGF vs plaquetas & 0.12 & 0.526 \\
\hline PIGF vs depuración de creatinina & -0.034 & 0.872 \\
\hline PIGF vs proteínas de 24 horas & 0.236 & 0.236 \\
\hline PIGF vs proteínas/creatinina & -0.449 & 0.225 \\
\hline sFlt-1/PIGF vs TAS & 0.157 & 0.406 \\
\hline sFlt-1/PIGF vs TAD & 0.042 & 0.824 \\
\hline sFlt-1/PIGF vs urea & -0.179 & 0.345 \\
\hline sFIt-1/PIGF vs creatinina & -0.116 & 0.54 \\
\hline sFIt-1/PIGF vs ácido úrico & 0.042 & 0.825 \\
\hline sFlt-1/PIGF vs plaquetas & -0.047 & 0.807 \\
\hline sFlt-1/PIGF vs depuración de creatinina & -0.051 & 0.808 \\
\hline sFlt-1/PIGF vs proteínas de 24 horas & -0.22 & 0.27 \\
\hline sFIt-1/PIGF vs proteínas/creatinina & 0.509 & 1.161 \\
\hline
\end{tabular}

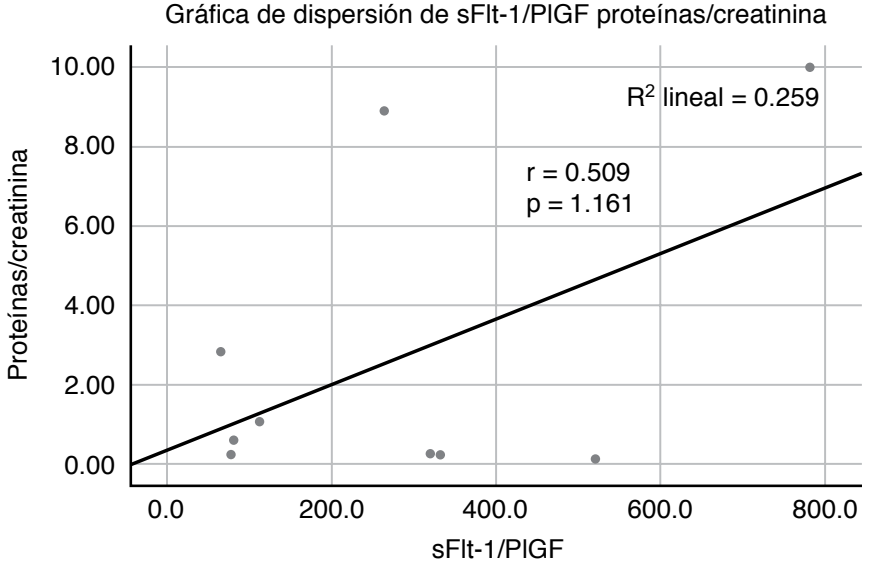

Figura 1: Se muestra el gráfico de dispersión y correlación del cociente sFlt-1/PIGF vs proteínas/creatinina, la cual es positiva pero no fue estadísticamente significativa.

2 fue de $31.3 \pm 6.5$ vs $28.6 \pm 6.0(p=0.1)$ y de semanas de gestación (SDG) de $30.1 \pm 5.1$ vs $28 \pm 4$ ( $p=0.7$ ). Existió una diferencia en los valores de sFlt-1, la cual fue de $5,907 \pm 4,823$ vs $1,332 \pm 675$ ( $p=0.001)$ y de PIGF 81 \pm 86 vs $398 \pm 299(p=0.001)$; el cociente de sFlt-1/PIGF fue de $234 \pm 397$ vs $4.5 \pm 2.9$ ( $p=0.005)$. Existió diferencia estadística en los promedios de las variables de laboratorio y bioquímicas entre los casos de preeclampsia y las mujeres sanas $(p<0.05)$. La tensión arterial sistémica (TAS) fue de $152 \pm 12$ vs $106 \pm 8$, la tensión arterial diastólica (TAD) fue de $66.4 \pm 6.7$, y el ácido úrico de 6.2 \pm 1.2 vs $4.1 \pm 8.3$, así como la TGO $28.14 \pm 26.5$ vs 21.13 \pm 10.39 ; la TGP $36.2 \pm 46.2$ vs $29.2 \pm 12.1$ y las proteínas en orina de 24 horas tuvieron un valor de 3,581 vs $217 y$ la creatinina sérica de $1.15 \pm 1.1$ vs $0.5 \pm 0.06$.

En el «grupo 1» hubo $33.3 \%$ de preeclampsia temprana (menos de 34 semanas de gestación) y $66.7 \%$ de preeclampsia tardía. En el «grupo 2» no hubo ningún caso de enfermedad hipertensiva asociada con el embarazo. Los criterios de severidad ocurrieron en $66.7 \%$ con crisis hipertensiva y en $33.3 \%$ con encefalopatía hipertensiva. La indicación de interrupción del embarazo fue la pérdida del bienestar fetal en $7.3 \%$ y en $90.2 \%$ existió deterioro materno como indicación de resolución del embarazo en el «grupo 1».

De las 42 mujeres del «grupo 1 », 30 casos tuvieron valores completos para realizar el análisis de correlación. Existió una correlación negativa entre los valores de sFlt-1 y urea, creatinina, proteínas de orina en 24 horas, TAS y TAD (Figuras 1 a 3). Sin embargo, la correlación fue pobre y no fue estadísticamente significativa, aunque existió una correlación positiva y estadísticamente significativa para ácido úrico. También hubo una correlación negativa entre los valores de PIGF en TAS y TAD. No existió correlación entre los valores cociente sFIt-1/PIGF y las variables medidas como TAS, TAD, etc. (Tabla 1). 


\section{DISCUSIÓN}

Diversos autores han definido la disfunción placentaria como un espectro de condiciones que incluyen preeclampsia, restricción del crecimiento intrauterino, desprendimiento de placenta, parto pretérmino y muerte fetal. ${ }^{6,8,9}$ Actualmente todas estas patologías conforman una entidad conocida como síndrome isquémico placentario. ${ }^{7}$ Dicho síndrome en su proceso fisiopatológico favorece el incremento de marcadores séricos en la circulación materna, derivado de una hipoxia placentaria y causado por un desbalance de los factores proangiogénicos/angiogénicos.

Sharon E. Maynard y otros autores ${ }^{3}$ han demostrado que la preeclampsia se asocia con una sFlt-1 circulante elevada. Un exceso de producción de sFlt-1 se origina en la placenta, y ha sido demostrado que el ARNm de sFlt-1 placentario está regulado por incremento en la preeclampsia y que los niveles caen dentro de las 48 horas posteriores al parto. Otros estudios demuestran que el sFlt-1 administrado exógenamente es suficiente para producir varios de los hallazgos clínicos y patológicos de preeclampsia en ratas, incluyendo hipertensión y endoteliosis glomerular con proteinuria. ${ }^{1}$ Sin embargo, estos mismos autores sugieren que los efectos sistémicos de sFlt-1 no requieren la presencia de la placenta, ya que la hipertensión y los cambios glomerulares ocurrieron tanto en ratas preñadas como no preñadas. Es posible que tanto la hipertensión como la proteinuria no dependan en su totalidad de los altos niveles de sFlt-1 y los bajos niveles de PIGF, aunque tradicionalmente se considera un efecto directo de sFlt-1 sobre el endotelio materno. ${ }^{10}$ Maynard también sugiere que el sFlt-1 actúa a través de su antagonismo tanto de VEGF como de PIGF, ya que su administración no produjo el fenotipo de preeclampsia en

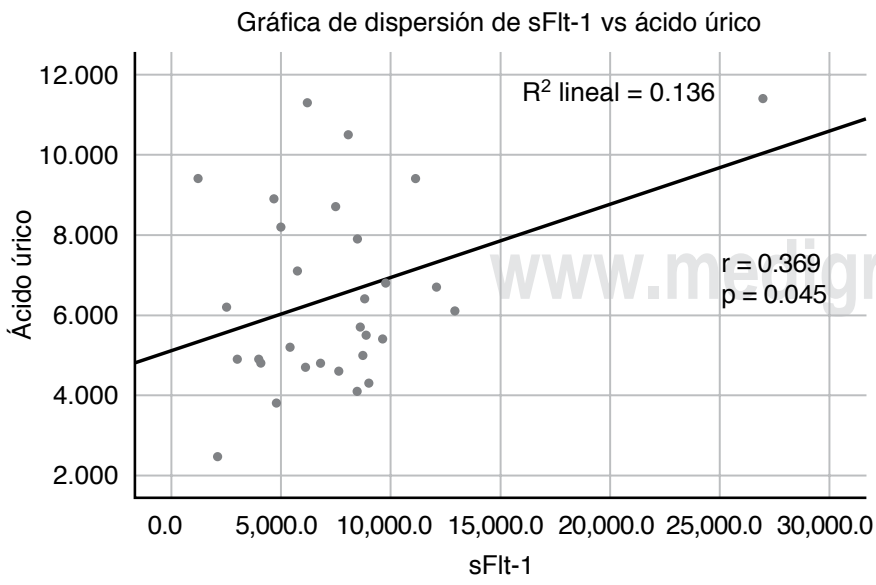

Figura 2: Se observa una correlación positiva en la gráfica de dispersión de sFlt-1 vs ácido úrico. Ésta fue la única variable que se obtuvo estadísticamente significativa.

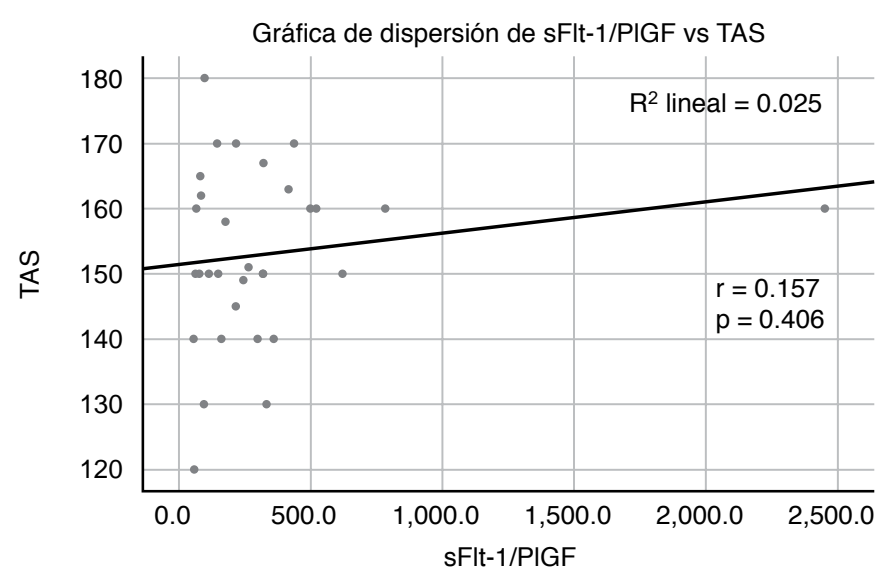

Figura 3: Aunque se observa una correlación positiva, la presión arterial sistólica no fue estadísticamente significativa.

ratas preñadas. Actualmente se han descrito una gran cantidad de mediadores que inducen hipertensión, proteinuria, elevación de transaminasas en pacientes con preeclampsia, es decir, el desbalance angiogénico no es el único mecanismo para explicar los hallazgos clínicos de severidad. Esto explica por qué no existe una correlación directa entre las mediciones que se realizaron en este estudio y los hallazgos clínicos y de laboratorio. ${ }^{1,9,10}$

\section{CONCLUSIÓN}

Este estudio confirma que es posible identificar un desbalance angiogénico en mujeres con preeclampsia severa, sin embargo, los marcadores angiogénicos no presentaron una correlación estadísticamente significativa con respecto a las variables clínicas y bioquímicas de preeclampsia con criterios de severidad. Se requiere realizar más estudios para valorar su utilidad en el ámbito clínico.

\section{BIBLIOGRAFÍA}

1. Purwosunu Y, Sekizawa A, Farina A, Wibowo N, Koide K, Okazaki S, et al. Evaluation of physiological alterations of the placenta through analysis of cell-free messenger ribonucleic acid concentrations of angiogenic factors. Am J Obstet Gynecol. 2008;198(1):124.e1-124.e7.

2. Cheng M, He P, Fu J. The relationship between circulating tissue transglutaminase, soluble fms-like tyrosine kinase-1, soluble endoglin and vascular endothelial growth factor in pre-eclampsia. J Hum Hypertens. 2016;30(12):788-793.

3. Maynard SE, Min J, Merchan J, Lim KH, Li J, Mondal S, et al. Excess placental soluble fms-like tyrosine kinase 1 (sFlt1) may contribute to endothelial dysfunction, hypertension, and proteinuria in preeclampsia. J Clin Invest [Internet]. 2003;111(5):649-658. Available from: http://www.jci.org/articles/ view/17189

4. Sibai BM, Taslimi MM, el-Nazer A, Amon E, Mabie BC, Ryan GM. Maternal-perinatal outcome associated with the syndrome of hemolysis, elevated liver enzymes, and low platelets in severe preeclampsia-eclampsia. Am J Obstet Gynecol. 1986;155(3):501-509. 
5. Zeisler H, Llurba E, Chantraine F, Vatish $M$, Staff $A C$, Sennström $\mathrm{M}$, et al. Predictive value of the sFIt-1:PIGF ratio in women with suspected preeclampsia. N Engl J Med. 2016;374(1):13-22.

6. de Jesus GR, de Jesus NR, Levy RA, Klumb EM. The use of angiogenic and antiangiogenic factors in the differential diagnosis of pre-eclampsia, antiphospholipid syndrome nephropathy and lupus nephritis. Lupus. 2014;23(12):1299-1301.

7. American College of Obstetricians and Gynecologists; Task Force on Hypertension in Pregnancy. Hypertension in pregnancy. Report of the American College of Obstetricians and Gynecologists' Task Force on Hypertension in Pregnancy. Obstet Gynecol. 2013;122(5):1122-1131.

8. Rana S, Powe CE, Salahuddin S, Verlohren S, Perschel FH, Levine RJ, et al. Angiogenic factors and the risk of adverse outcomes in women with suspected preeclampsia. Circulation. 2012;125(7):911-919.

9. Kleinrouweler CE, Wiegerinck MM, Ris-Stalpers C, Bossuyt PM, van der Post JA, von Dadelszen P, et al. Accuracy of circulating placental growth factor, vascular endothelial growth factor, soluble fms-like tyrosine kinase 1 and soluble endoglin in the prediction of pre-eclampsia: a systematic review and metaanalysis. BJOG. 2012;119(7):778-787.
10. Agrawal S, Cerdeira AS, Redman C, Vatish M. Meta-analysis and systematic review to assess the role of soluble fms-like tyrosine kinase-1 and placenta growth factor ratio in prediction of preeclampsia: the SaPPPhirE study. Hypertension. 2018;71(2):306-316.

Patrocinios: Ninguno.

Relación de conflicto de intereses: Sin conflicto de intereses.

\section{Correspondencia:}

Luis Alberto Ruiz Marines

Montes Urales Núm. 800,

Col. Lomas de Virreyes,

Del. Miguel Hidalgo, 11000,

Ciudad de México, México.

E-mail:medics_luis@hotmail.com 